Postgrad. Med. J. (1965), 41, 776

Current Surveys

\title{
ADOLESCENT COXA VARA
}

\author{
P. R. SHIRES, F.R.C.S.
}

St. Thomas's Hospital, London*

ONE justification for a further paper on slipping of the upper femoral epiphysis would be the all too frequent delay in diagnosis of this condition. We now have a tenable theory of its cause, and there has been much useful re-evaluation of its operative treatment, but in the years ahead many degenerate hips resulting from severe old adolescent coxa vara will continue to be seen.

The label invites confusion, for the condition is, of course, essentially prepubertal rather than adolescent. The onset of puberty has always been a variable feast, and fashions change; at the time of writing, the dangerous period in girls (for their hips) may have come and gone by the age of ten or eleven, and once menstruation has started it is most unlikely that a femoral epiphysis will begin to displace. Boys begin adolescence later, but certainly the average age of those affected is younger than it was a generation ago. An endocrine background which has been presumed for many years has still not been fully explained, and the relationship of sex hormone to the ossification of the cartilaginous epiphysis has been questioned: it may be that circulating sex hormone at puberty simply inhibits the anterior pituitary's production of growth hormone and thus indirectly the elongation of the epiphyseal cartilage. (Harris, 1950). The Frölich physique is associated with one third of the patients, but mechanical factors are probably more concerned than hormonal ones in the shearing stress through an oblique disc; many of the children are tall, or unusually heavy, or both (Burrows, 1957). It has been postulated, in simple terms, that when the ratio of sex hormone to growth hormone is reduced, from relative lack of the one or relative excess of the other, then the cartilage is especially vulnerable. A child's build, however, may mislead, since a further one-third are children of normal prepubertal physique, and this is one diagnostic pitfall.

\section{Diagnosis}

Of the two main kinds of presentation, the acute sudden slip is less likely to be overlooked. Some physical activity may be cut short by a dramatic incident, and the child is painfully unable to lift the affected leg, which is short and externally rotated. The signs are similar to those of a fractured femoral neck, and X-rays show that the upper femoral epiphysis has migrated, by half its diameter or more, downwards and backwards off the femoral neck. The commoner presentation of gradual, or minor repeated slipping, which accounts for nearly three quarters of these patients, is the one which can evade early diagnosis. Children of this age are often secretive about minor aches in the groin or knee, and a limp may disappear when a temporary irritability of the hip clears up; parents delay seeking advice, and console themselves with the non-diagnoses of 'growing pains' or 'rheumatism'; and, when advice is sought, the site of referred pain sometimes distracts attention from the hip joint to a normal knee. The local hip signs, in a trivial degree of slipping, may amount to no more than a small loss of abduction and internal rotation. Finally, and regrettably, the child may be passed as normal after an anteroposterior film of the hip shows no apparent slip, when a lateral film would clearly reveal the earlier, posterior displacement (Fig. 1). X-ray signs have been devised by Trethowan and Capener (Fig. 2) to assist diagnosis of the early stage; the more advanced chronic slip is obvious clinically, in the gross loss of abduction and internal rotation, and from the X-rays (Fig. 3).

\section{Treatment}

The treatment is surgical, for it is not feasible to take a child off weight-bearing until puberty should start, in the hope of forestalling further epiphyseal shift. Nor has hormonal tinkering been found to have any value. Preliminary skin traction may succeed in reducing, or partially reducing, a sudden slip, especially if an element of gradual internal rotation can be introduced. Traction for the chronic slip is useless, but may comfort the patient awaiting surgery. It should be remembered that the epiphysis may be displaced in the opposite hip during bed treatment, for the weight of the leg tending to externally rotate can cause sufficient shearing stress.

The bugbear of avascular necrosis threatens all kinds of further treatment, for surgical interference may tilt the balance of an already precarious blood supply to the epiphysis. Coxa vara can be avoided or corrected, but the femoral head may die and the joint wear out at an early age.

The trivial gradual slip which can be left unreduced, without incongruity of the hip joint, offers the best kind of result. Simple internal fixation, to check further slip before the epiphysis

*Present address :

Royal Surrey County Hospital, Guildford. 


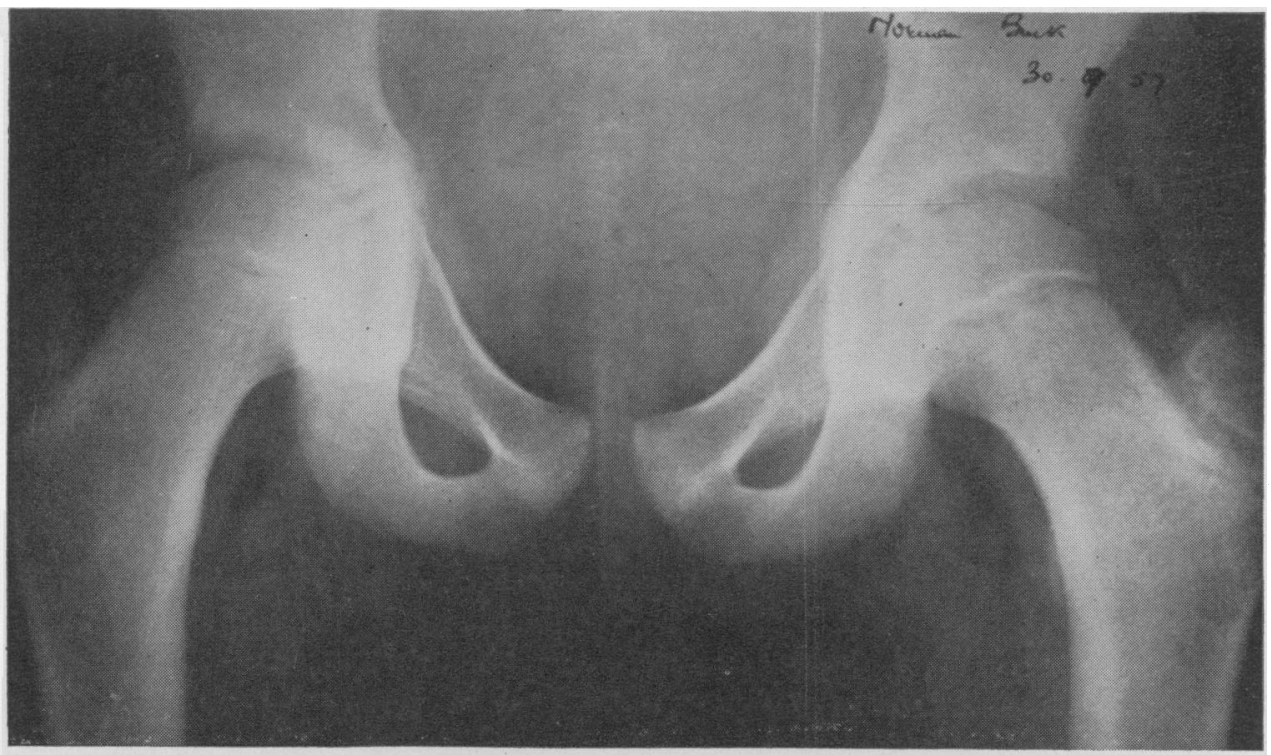

(a)

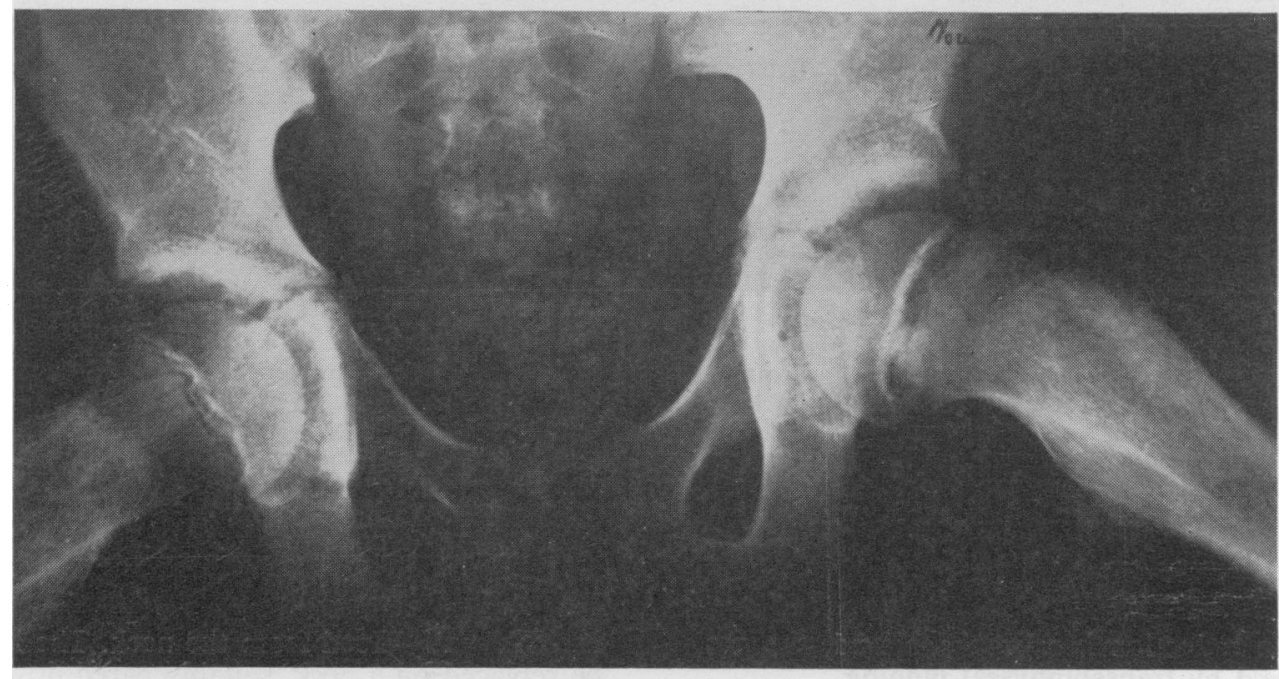

(b)

FIG. 1.-The AP view could be passed as normal.

The displacement is clear in the lateral film.
$\begin{array}{ll}\text { (a) AP } & \text { (b) Lateral. }\end{array}$
(a) AP

fuses, is all that is required. Moore's pins or their variants are introduced in the manner of pinning a transcervical fracture, and imageintensification radiography reduces this to an operative triviality. These pins seem to avoid the avascular necrosis which one saw after insertion of the trifin nail, which not only cuts across vascular pathways in the neck by its bulk, but which also required considerable bludgeoning to traverse the young, hard bone. Such trauma must imperil the head.
The sudden slip needs reduction comparatively urgently. If traction fails, it is reasonable to attempt manipulative reduction by internally rotating the hip, but this should be done with almost timorous gentleness, for the risks are great. If for any reason treatment has been delayed for a day or two, the all-important subsynovial retinacular vessels to the head adaptively shorten, and in such cases it is probably better to withhold closed manipulation in favour of an open procedure on the femoral neck (see below). Once an 


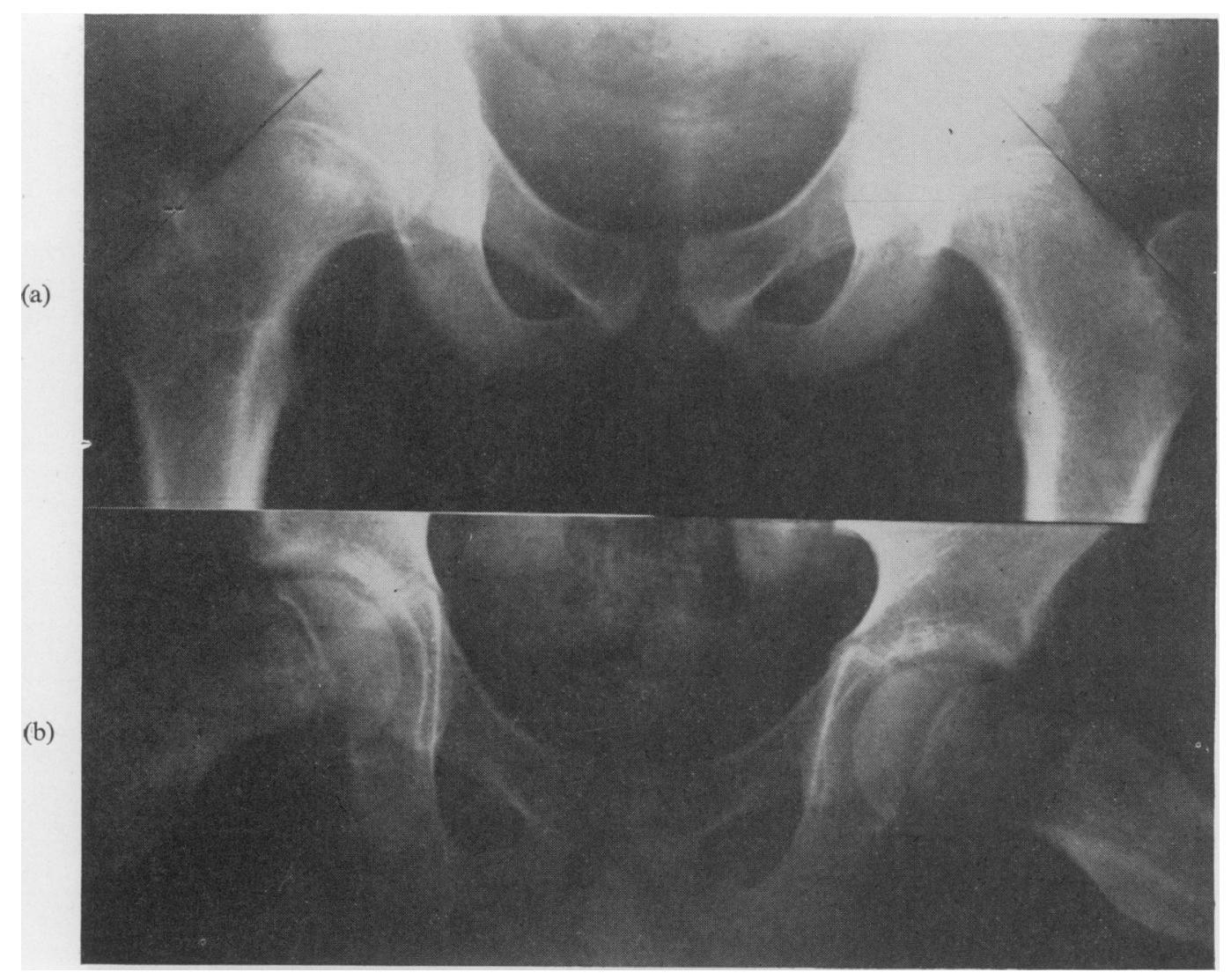

FIG. 2.- Trethowan's line, drawn through the upper border of the femoral neck, should "cut off" a substantial portion of the head.

Capener's sign : in the AP film of the normal hip, the acetabulum overshadows a triangle of metaphysis on the medial side next to the epiphysis. When the head has slipped, the metaphysis appears wholly outside the acetabulum.

(a)AP-Left normal. Right abnormal. (b) Lateral confirms displacement.

acceptible reduction has been achieved, the position is hell by transepiphyseal pinning.

The severe chronic slip. When diagnosis has been too late, it is often clear from the X-rays that, even if it were possible to impale the epiphysis by pins passing through the femoral neck, too much deformity would be left. Cervical wedge osteotomy would seem to be an obvious method of correction, and has been practised in certain centres for many years; so frequently, however, did necrosis of the head follow, that it was discredited in this country and apparently abandoned. Recently it has been revised by Dunn (1964), who is being copied with enthusiasm. Through a lateral approach to the hip, the vascular synovium and subsynovial tissues, running posteriorly up the femoral neck to the epiphysis, are carefully defined and preserved when the head itself is gently freed from the neck. The head can be replaced on a neck, suitably pared down, without tension in the posterior retinacular vessels, and it is fixed with Moore's pins. The early results in Dunn's hands have been encouraging, but it is by no means certain that this operation is a safe procedure for universal practice.

If, indeed, cervical osteotomy can be rediscovered in a way that drastically cuts the number of avascular femoral heads, it must be a superior treatment to subtrochanteric wedge osteotomy. This operation has two objects: deformities at the hip joint are compensated, and the epiphyseal cartilage is made more "horizontal", in the hope of preventing further slip. Removal of an anterolaterally based wedge at subtrochanteric level may realign the head well and provide a hip with fair function, but gross slips can defy the most 


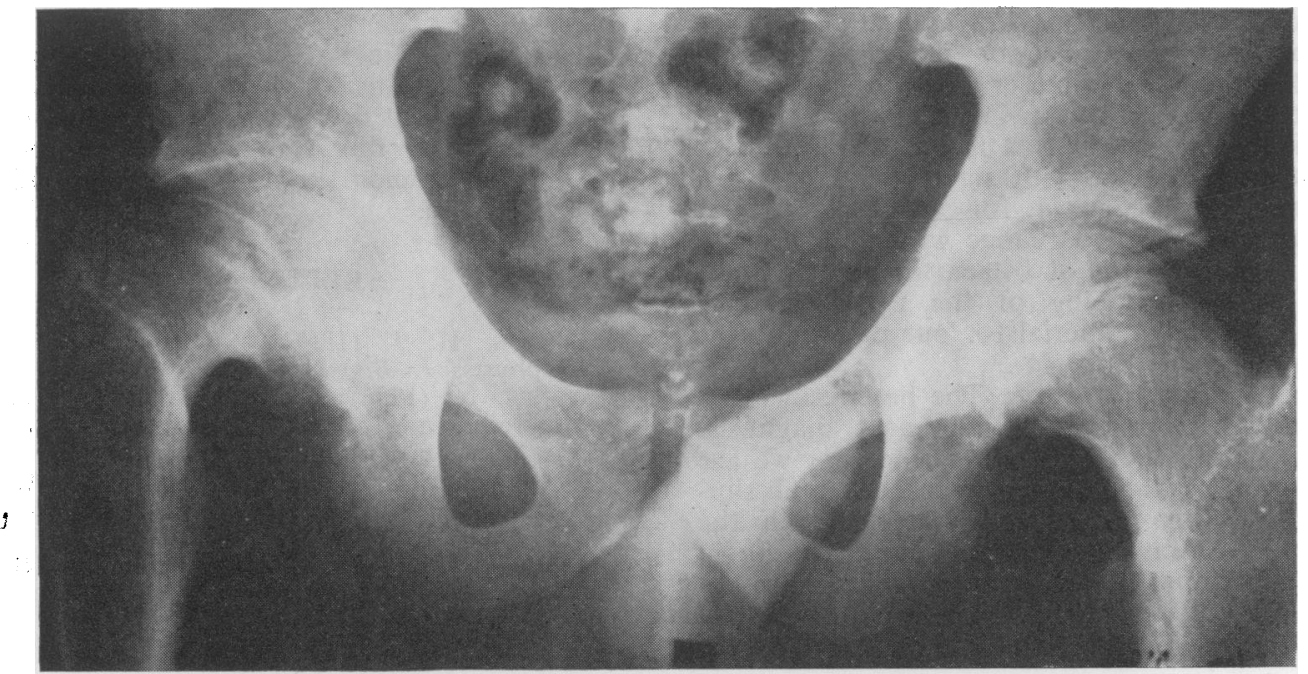

(a)

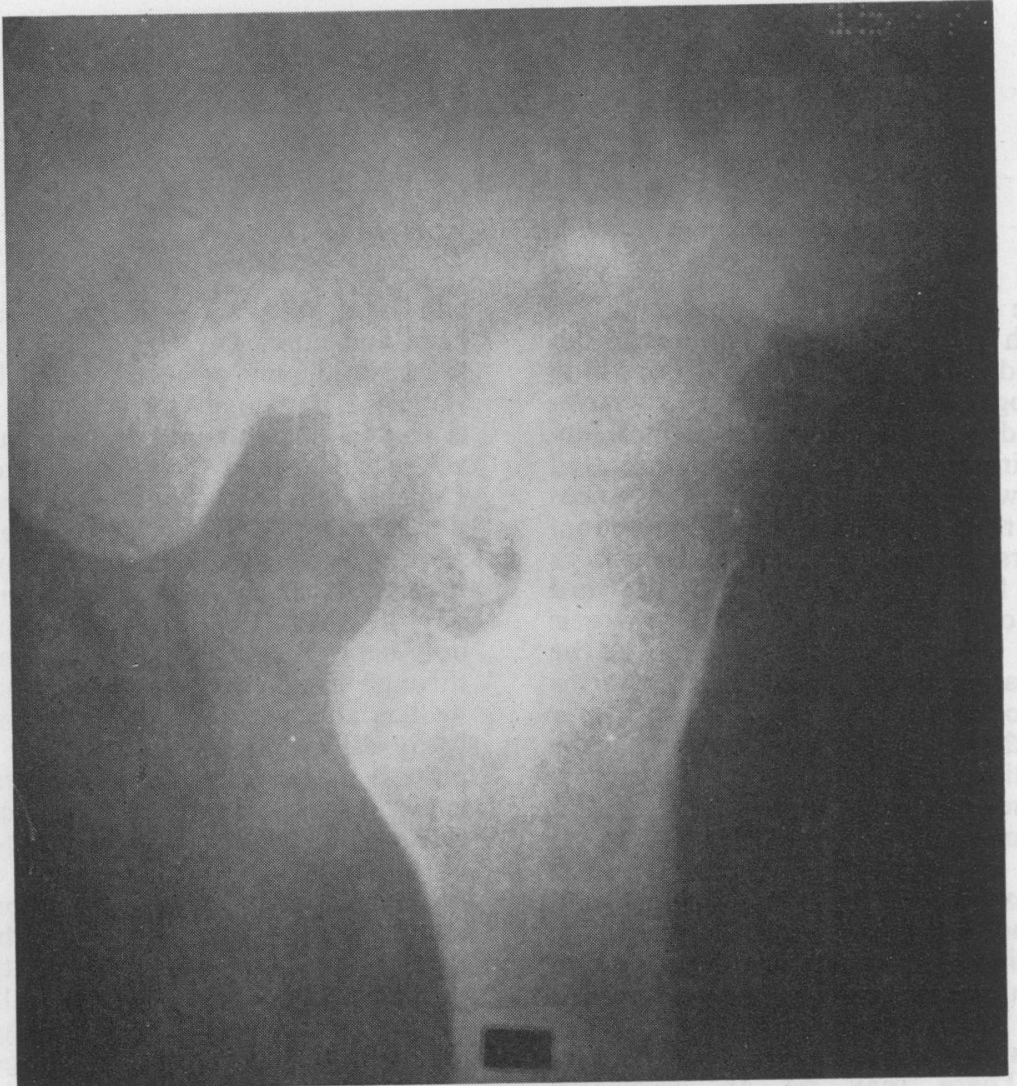

(b)

FIG. 3.-Severe chronic slip.
(a) AP
(b) Lateral. 
painstaking efforts in geometry and surgery; compensation is often short of the diagrammatic textbook ideal. Subtrochanteric osteotomy remains the best hope of salvage when a badly distorted epiphysis has closed. Indeed it should be offered. The untreated patient with significant coxa vara may have little concern for some years; but premature degenerative change will surely follow, and the bizarre forms of osteoarthritis which are seen after past slipping of the upper femoral epiphysis are poor material for our present forms of hip surgery.

The conclusion is obvious. The best results and the simplest treatment follow early diagnosis. Vigilance cannot be overdone. Sometimes we are easily forewarned. Children with unilateral slipping are told to report any symptoms in the other leg. Dickensian fat boys, found lurking in hospital corridors, have been inveigled into an examination of their hips, and discovered with early 'slips'. The background is often much less suggestive, for in twelve-year-old children minor trauma and transient lower limb pains are not uncommon. But awareness may breed suspicion that a hip in this age group could be faulty, and such suspicion should be proved or disproved with full care.

\section{REFERENCES}

Burrcws, H. J. (1957): Slipped Upper Femoral Epiphysis, J. Bone Jt. Surg., 39, 641.

CAPENER, N. (1956): Reconstructive Surgery of the Hip Joint. In 'Modern Trends in Orthopaedics,' p. 4. 2nd Series, ed. Sir H. Platt. London: Butterworths.

DunN, D. M. (1964): The Treatment of Adolescent Slipping of the Upper Femoral Epiphysis, J. Bone Jt. Surg.. 46B, 621 .

HARRIS, W. R. (1950): The Endocrine Basis for Slipping of the Upper Femoral Epiphysis, J. Bone Jt. Surg., 32B, 5.

\title{
ACHALASIA OF THE CARDIA
}

\author{
Michael Bates, F.R.C.S. \\ Thoracic Surgeon, North Middlesex Hospital, London, N.18.
}

Achalasia of the cardia is a distressing condition which affects both sexes equally, and patients from childhood to old age. Achalasia is becoming increasingly recognised due to great improvements in the radiological techniques which demonstrate by cineradiography the complicated mechanism of swallowing and the disturbances which occur at the gastro-oesophageal junction. It is now universally accepted that achalasia is the correct term for this condition and the word cardiospasm is no longer used. However there is still considerable difference of opinion as to the best form of treatment and therefore this seems a suitable time to review the subject, particularly in relation to the complications, both of the untreated condition and also of the various treatments at present in use.

Adams and Trounce from Guy's Hospital wrote a classic article on this subject in 1961 when they went very extensively into the aetiology and pathology of the condition and analysed the results in 85 patients. They divide the condition into three clinical stages and in stage 1 there is often a history of sudden onset of marked dysphagia, frequent regurgitation and oesophageal pain. There may be an associated emotional disturbance although Flavell (1963) thinks this very unlikely particularly in children. The patient can become anaemic but little oesophageal dilatation occurs in the early stage. In stage 2 the oesophagus becomes markedly dilated and can hold a very considerable quantity of food and fluid. There is no pain and little regurgitation and any weight which has been lost in stage 1 is regained. An adequate quantity of food is forced through into the stomach purely by the weight of food which lies above it. After about 15 years the 3 rd stage of mega-oesophagus has developed with a sigmoid loop on the diaphragm, severe weight loss, hypertrophic pulmonary osteoarthropathy, polyarthritis and very occasionally death from starvation. Barrett (1964) does not believe that the disease necessarily passes through these three stages and has patients whom he has followed up for 30 years where the fusiform dilatation of stage 2 , or cucumber oesophagus as he calls it, remains static. Many cases, generally old people, are diagnosed with a short history and a megaoesophagus found on the first $\mathrm{X}$-ray.

The aetiology of this condition has never been convincingly explained although all writers are agreed that there is a degeneration or even congenital absence of ganglion cells in Auerbach's myenteric plexus in the oesophageal muscle wall. Whether this is a primary congenital absence or a secondary degeneration due to many years of food stagnation with fibrosis of the oesophageal wall is not at all clear. It is difficult to understand why a patient with congenital absence of ganglion cells in his oesophageal wall should not develop achalasia until old age, and so it may be that 